\title{
Barriers and enablers of implementing gestational weight gain guidelines in GP-led shared maternity care: a qualitative study
}

\author{
R. Walker ${ }^{1}$, T. Choi $^{1}$, K. Alexander ${ }^{2}$, D. Mazza ${ }^{2}$ and H. Truby ${ }^{1}$ \\ ${ }^{1}$ Department of Nutrition, Dietetics and Food, School of Clinical Sciences, Monash University, Level 1, 264 Ferntree \\ Gully Road, Notting Hill, VIC 3168, Australia and Department of General Practice, School of Primary and Allied \\ Health Care, Monash University, 246 Clayton Road, Clayton, VIC 3168, Australia.
}

Approximately fifty percent of women gain too much weight during pregnancy ${ }^{(1)}$, increasing pregnancy risk ${ }^{(2)}$ and perpetuating the cycle of overweight and obesity ${ }^{(3)}$. With approximately 1.7 million consultations each year ${ }^{(4)}$, shared maternity care led by general practitioners (GPs) is a crucial component of antenatal care in Australia ${ }^{(5)}$. Little is known about the gestational weight gain (GWG) advice women receive in shared maternity care. Therefore, we aimed to explore GPs' perceptions, barriers and enablers implementing GWG guidelines.

General Practitioners $(n=20)$ who provide shared maternity care from metropolitan and regional Victoria (Australia) were recruited via postal invitation to participate in a semi-structured interview. Questions were informed by the Theoretical Domains framework $(\mathrm{TDF})^{(6)}$ that explores barriers and enablers of guideline implementation. Interviews were transcribed verbatim and transcripts were analysed by two researchers using deductive thematic analysis ${ }^{(7)}$. NVivo Version 11 (QSR International) was used in the analyses and ethics approval was obtained from Monash University (REF: 7799).

Key themes regarding GPs' awareness, knowledge and application of GWG guidelines aligned with the TDF. GPs were aware of the importance of optimal GWG for positive maternal and foetal outcomes however, other pregnancy-related issues were given priority in short consultations. Enablers were practitioner-based and included GPs' strong sense of their professional role to provide GWG advice, and ongoing and trusting relationships with women through and beyond pregnancy. Barriers were mostly health system-based with limited time, remuneration, and restrictive referral pathways being limiting factors.

There is an urgent need to support GPs to provide GWG advice in accordance with current national guidelines ${ }^{(8)}$. Solutions lie in changes to policy and funding within the health-system so that longer shared care consultations are remunerated, and referrals to dietitians and other allied health professionals are accessible to women who require additional support to optimise GWG beyond what their GP can offer.

1. Goldstein R, Abell S et al. (2017) JAMA 317, 2207-2225.

2. Cheney K, Farber R et al. (2018) Med J Aust 208, 119-125.

3. Melzer K \& Schutz Y. (2010) Int J Obes 34, S44-S52.

4. Wong C, Gordon J et al. (2016) Aust Fam Phys 45, 538-541.

5. Brodribb W. (2014) MJA 201, 626-267.

6. Cane J, O'Connor D et al. (2012) Implement Sci 7, 17 pages.

7. Atkins L, Francis J et al. (2017) Implement Sci 12, 18 pages.

8. Walker R, Mazza D et al. (2018) Aust J Prim Health 24, 4-8.

Main supervisor of PhD student, Ruth Walker: Professor Helen Truby, Department of Nutrition, Dietetics and Food, School of Clinical Sciences, Monash University, Notting Hill, Victoria, Australia.Email: helen.truby@monash.edu 